\title{
Up-regulation of BLT2 is critical for the survival of bladder cancer cells
}

\author{
Ji-Min Seo ${ }^{1}$, Kyung-Jin Cho ${ }^{1}$, Eun-Young Kim ${ }^{1}$, \\ Man Ho Choi ${ }^{2}$, Bong Chul Chung ${ }^{2}$ \\ and Jae-Hong $\mathrm{Kim}^{1,3}$ \\ ${ }^{1}$ School of Life Sciences and Biotechnology \\ Korea University \\ Seoul 136-701, Korea \\ ${ }^{2}$ Life Sciences Division \\ Korea Institute of Science and Technology \\ Seoul 136-791, Korea \\ ${ }^{3}$ Corresponding author: Tel, 82-2-3290-3452; \\ Fax, 82-2-927-9028; E-mail, jhongkim @korea.ac.kr \\ DOI 10.3858/emm.2011.43.3.014
}

Accepted 20 January 2011

Available Online 20 January 2011

Abbreviations: HETE, hydroxyeicosatetraenoic; LO, lipoxygenase; $\mathrm{LTB}_{4}$, leukotriene $\mathrm{B}_{4}$; NOX, NADPH oxidase; ROS, reactive oxygen species

\begin{abstract}
The incidence rates of urinary bladder cancer continue to rise yearly, and thus new therapeutic approaches and early diagnostic markers for bladder cancer are urgently needed. Thus, identifying the key mediators and molecular mechanisms responsible for the survival of bladder cancer has valuable implications for the development of therapy. In this study, the role of BLT2, a receptor for leukotriene $\mathrm{B}_{4}\left(\mathrm{LTB}_{4}\right)$ and 12(S)-hydroxyeicosatetraenoic acid (HETE), in the survival of bladder cancer 253J-BV cells was investigated. We found that the expression of BLT2 is highly elevated in bladder cancer cells. Also, we observed that blockade of BLT2 with an antagonist or BLT2 siRNA resulted in cell cycle arrest and apoptotic cell death, suggesting a role of BLT2 in the survival of human bladder cancer 253J-BV cells. Further experiments aimed at elucidating the mechanism by which BLT2 mediates survival revealed that enhanced level of reactive oxygen species (ROS) are generated via a BLT2-dependent up-regulation of NADPH oxidase members NOX1 and NOX4. Additionally, we observed that inhibition of ROS generation by either NOX1/4 siRNAs or treatment with an ROS-scavenging agent results in apoptotic cell death in 253J-BV bladder cancer cells. These results demonstrated that
\end{abstract}

\begin{abstract}
a 'BLT2-NOX1/4-ROS' cascade plays a role in the survival of this aggressive bladder cancer cells, thus pointing to BLT2 as a potential target for anti-bladder cancer therapy.
\end{abstract}

Keywords: biological markers; cell survival; LTB4R2 protein, human; NADPH oxidase; reactive oxygen species; urinary bladder neoplasms

\section{Introduction}

Bladder cancer is the second most common genitourinary malignant disease and the number of diagnosed cases is increasing annually in the United States (Jemal et al., 2008). Bladder cancer can be divided into two groups (superficial or invasive) depending on the pathologic stage. Approximately $90 \%$ of malignant tumors arising in the urinary bladder are epithelial origin, the majority being transitional cell carcinomas (TCCs). Of all newly diagnosed cases of TCCs, about $70 \%$ present as superficial tumor (stages Tis, Ta, and T1), but as many as $50-70 \%$ of those superficial tumor will recur and $10-20 \%$ will progress to more invasive and aggressive tumor (stages T2-4). The high rate of recurrence and the poor prognosis of invasive urothelial carcinoma emphasize the necessary for the development of effective targeted therapies (Cordon-Cardo, 2008; Kaufman et al., 2009).

Arachidonic acid (AA) is released from membrane phospholipids by cytosolic phospholipase $\mathrm{A}_{2}\left(\mathrm{CPLA} \mathrm{A}_{2}\right)$ and is further metabolised via the cyclooxygenase (COX), lipoxygenase (LO) and cytochrome p450dependent pathways. Among these, recent studies have revealed potential roles of the 5-, 12-, and 15-lipoxygenase (LO) pathways in cancer progression. For example, 5- and 12-LO are overexpressed in bladder cancer tissues and LO inhibitors inhibited growth of bladder cancer and induced apoptosis (Yoshimura et al., 2003; Hayashi et al., 2006). In accordance with the suggested role of 12-LO in cancer, 12(S)-hydroxyeicosatetraenoic acid (12(S)HETE), a 12-LO pathway metabolite, was shown to influence tumor progression (Nie et al., 2006). Likewise, leukotriene $\mathrm{B}_{4}\left(\mathrm{LTB}_{4}\right)$, one of the 5-LO pathway metabolites, was recently shown to stimulate cell proliferation via ERK signalling pathways in several types of cancer cells, including pancreatic 
and colon cancer cells (Hennig et al., 2004; Tong et al., 2005). In addition, the $\mathrm{LTB}_{4}$ receptor antagonist LY293111 inhibited the growth of human pancreatic cancer cells and induced apoptosis of lymphoma cells (Zhang et al., 2005; Tong et al., 2007). These results suggest that $\mathrm{LTB}_{4}$ and its receptors may play cancer-promoting roles during cancer progression. Two G-protein-coupled $\mathrm{LTB}_{4}$ receptors have been cloned and characterised: BLT1 and BLT2. BLT1 is a high-affinity receptor specific for $L_{1}{ }_{4}$, whereas BLT2 is a low-affinity receptor that also binds 12(S)-HETE (Tager and Luster, 2003). To date, most studies of $\mathrm{LTB}_{4}$ receptors have focused on BLT1. By contrast, very little biological functions of BLT2 has been identified although recent reports suggest that it is likely to be involved in various capacities of cancer cells, thus potentially contributing to cancer progression (Hennig et al., 2008; Choi et al., 2010; Kim et al., 2010b).

In the present study, we found that BLT2 contributes to survival signalling in 253J-BV bladder cancer cells. We also found that NOX1- and NOX4dependent ROS are essential downstream components of the BLT2-mediated survival signalling that protects cells against apoptotic cell death. Therefore, we suggest that a 'BLT2-NOX1/4-ROS'linked cascade is a novel pathway for the survival of bladder cancer cells and offers a new target for bladder cancer therapy.

\section{Results}

\section{BLT2 inhibition by LY255283 or siRNA induces apoptosis in 253J-BV bladder cancer cells}

To investigate a possible role of $B L T 2$, a receptor that binds $\mathrm{LTB}_{4}$ and $12(\mathrm{~S})$-HETE, in bladder cancer cells, the levels of BLT2 mRNA were first examined in five different human bladder cancer cell lines and immortalised uroepithelial SV-HUC-1 cells. Realtime PCR analysis showed that most bladder cancer cells express higher levels of BLT2 mRNA than control SV-HUC-1 cells (Supplemental Figure S1A). In contrast, BLT1 mRNA levels are not enhanced except in 253J-BV cells. Also, the levels of BLT2 ligands, $\mathrm{LTB}_{4}$ and $12(\mathrm{~S})$-HETE, are markedly enhanced in 253J-BV and 253J cells compared with control cells, as shown in Supplemental Figure S1B. Together, both BLT2 and its ligands appear to be upregulated in bladder cancer cells and thus suggest a potential role of BLT2 signalling in bladder cancer progression.

Next, we examined whether BLT2 blockade affects growth or survival of bladder cancer cells. As shown in Figure 1, treatment with a BLT2 anta- gonist LY255283, but not with a BLT1 antagonist U75302, induced cell cycle arrest and subsequent apoptotic cell death in 253J (B) and 253J-BV bladder cancer cells (C). In contrast, SV-HUC-1 immortalised control cells were not affected by either LY255283 or U75302 (A). Similarly, cell viability as analysed by the MTT assay was remarkably diminished by treatment with LY255283 for $48 \mathrm{~h}$ in $253 \mathrm{~J}$ cells (Figure 1B right) and 253JBV cells (Figure $1 \mathrm{C}$ right) compared with the control cells (Figure 1A right). Consistent with above results, treatment with LY255283 caused morphological changes, membrane blebbing and detachment at $48 \mathrm{~h}$ (Figure 1D). In contrast, treatment with a BLT1 antagonist U75302 did not affect cell morphology or survival (Figure 1D). To further analyse the apoptosis-inducing effects by BLT2 inhibition, we next performed western blot analysis and a DNA fragmentation assay in 253J-BV cells (Figures $1 \mathrm{E}$ and $1 \mathrm{~F}$ ). PARP cleavage and activation of Bax are important markers of apoptosis. Clearly, LY255283 caused apoptosis by activated PARP, reduced procaspase-9, and elevated Bax activity (Figure 1E). Furthermore, when cells were treated with LY255283 for $48 \mathrm{~h}$, we observed significant DNA ladder formation, which is a characteristic of apoptosis (Figure 1F), thus together suggesting that BLT2 is somehow necessary for bladder cancer cell survival.

To further analyse the contribution of BLT2 to bladder cancer survival, we performed siRNA knockdown of BLT2 in 253J-BV cells. As shown in Figures $2 \mathrm{~A}$ and $2 \mathrm{~B}, \mathrm{BLT} 2$ siRNA was effective for the selective knockdown of BLT2 mRNA levels and had no effect on BLT1 mRNA levels. Similar to the results observed using LY255283, knockdown of BLT2 induced cell cycle arrest at sub-G1 to a level comparable to that of the cells treated with LY255283 (Figure 2C). We found that Hoechst 33258 staining of 253J-BV cells were transfected with BLT2 siRNA revealed a remarkable nuclear DNA condensation, indicating apoptosis (Figure 2D). Results shown in Figure 2E, we observed that knockdown of BLT2 using siRNA induced cleavage of PARP. Together, these results suggest that BLT2 signalling is necessary for 253J-BV bladder cancer cell survival.

\section{BLT2 mediates pro-survival via DPI-sensitive NADPH oxidase}

Elevated oxidative status has been found in many cancer cells, and ROS are suggested to play key roles in cancer progression (Wu, 2006). We previously demonstrated that NOX1-mediated generation of ROS lies downstream of BLT2 (Woo et al., 

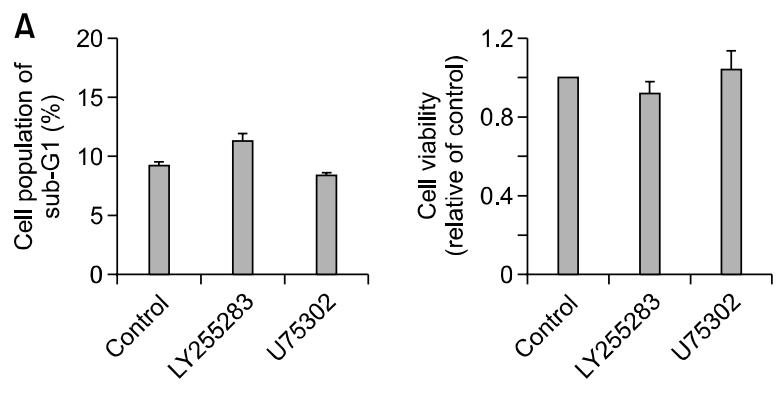

D
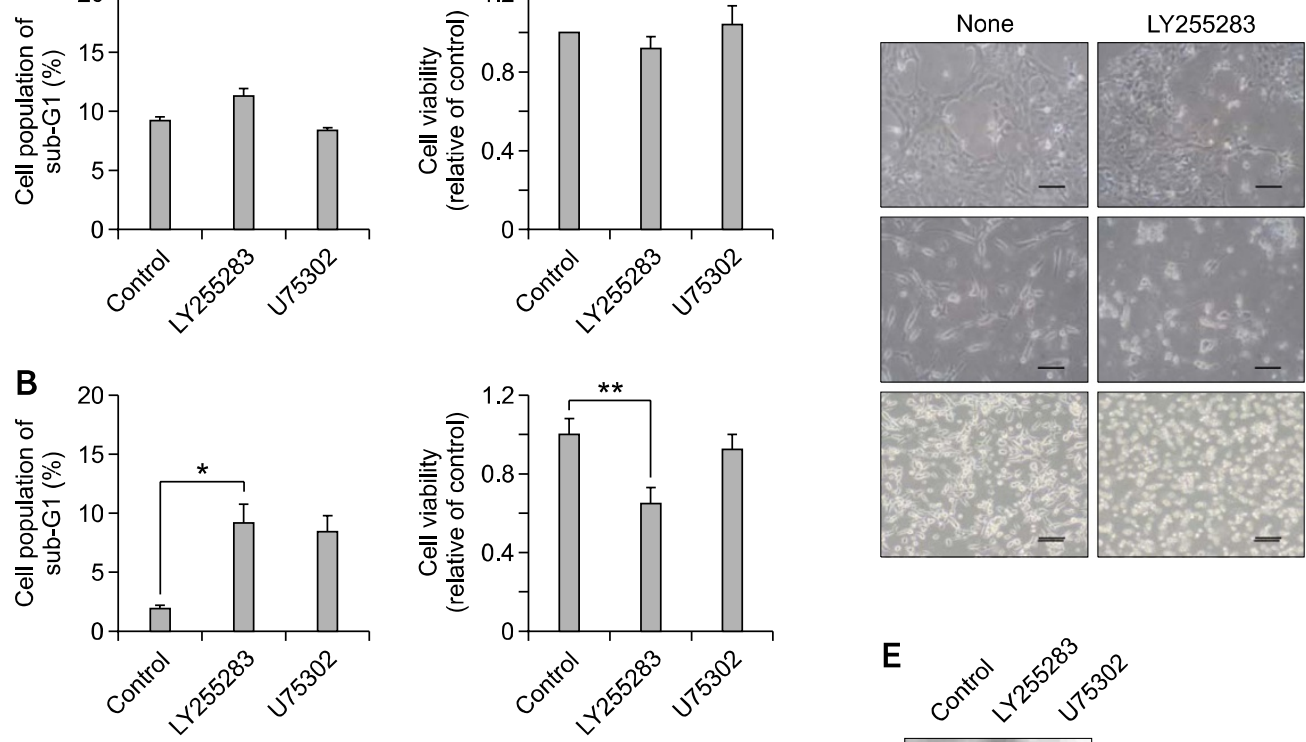

$\mathrm{U} 75302$
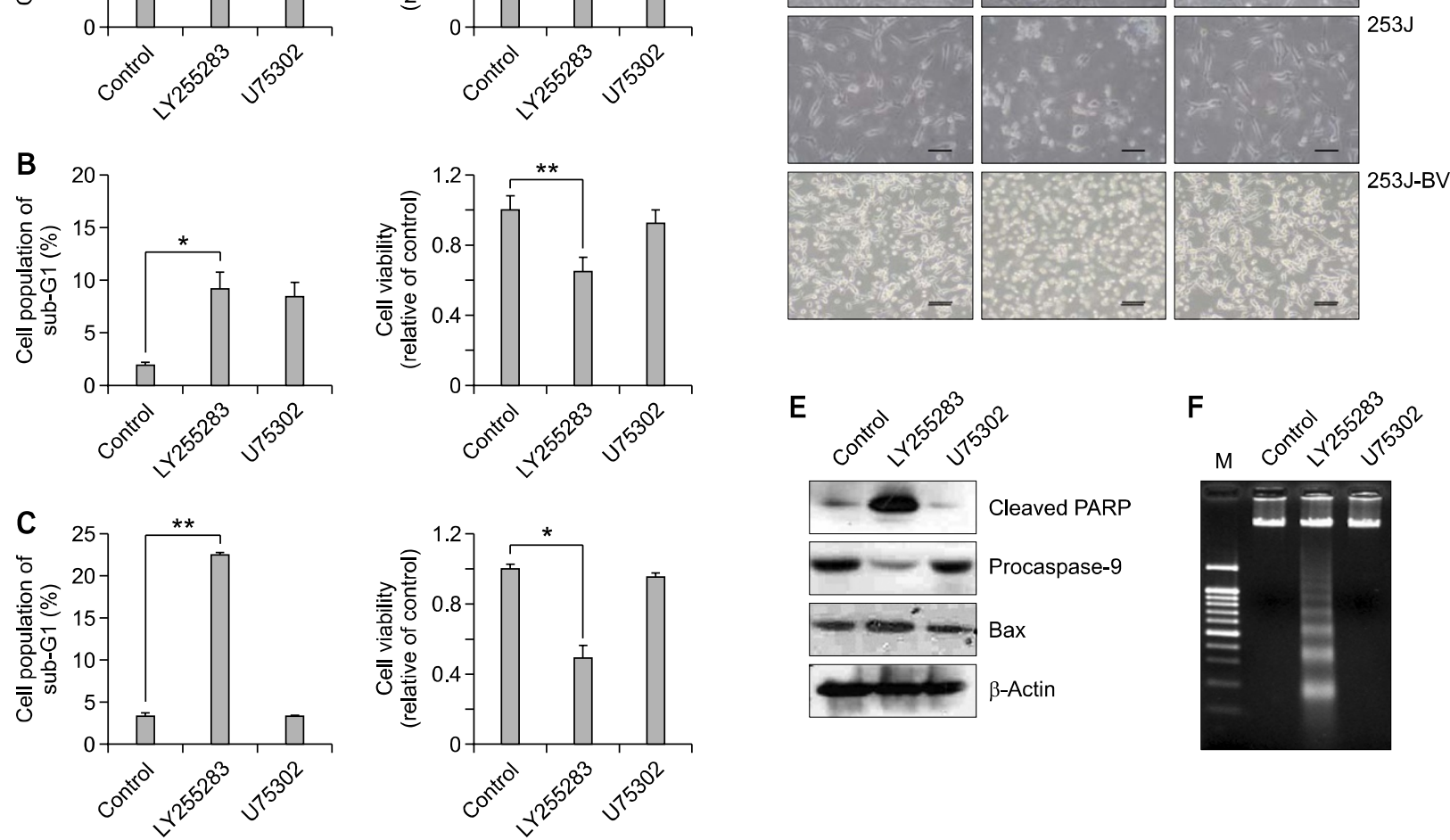

Figure 1. Effects of BLT2 antagonist LY255283 on the survival of 253J-BV cells. Treatment with LY255283 induced cell cycle arrest and subsequent apoptotic cell death in the bladder cancer cell lines 253J (B) and 253J-BV (C), but, not SV-HUC-1 cells (A). Cells were starved with $0.5 \%$ FBS medium for $12 \mathrm{~h}$, and then cells were treated with the indicated concentration of each antagonist. Cell cycle distribution ( $A, B$ and $C$ left panels) was measured by flow cytometry. Cells were treated with LY255283 $(10 \mu \mathrm{M})$ or U75302 $(1 \mu \mathrm{M})$ for $42 \mathrm{~h}$. Cell viability (A, B and C right panels) was detected by MTT assay (as described in the Supplemental material and methods) after treatment with LY255283 and U75302 for $48 \mathrm{~h}$. Data indicate the means \pm S.D. of three independent experiments $\left({ }^{*} P<0.01,{ }^{* *} P<0.001\right)$. (D) LY255283-induced cell death was visualised using an Olympus BX51 microscope at $\times 100$ magnification. Bar, $100 \mu \mathrm{m}$. (E) LY255283 induced PARP cleavage and activation of caspase-9 in 253J-BV cells. The level of pro-apoptotic protein Bax was increased significantly when cells were treated with LY255283 for $48 \mathrm{~h}$. (F) LY255283 caused internucleosomal DNA fragmentation in 253J-BV cells. The detail apoptotic analysis is described in the Supplemental material and methods.

2002; Choi et al., 2008; Kim et al., 2010a). This led us to examine the possible role of ROS in the survival signalling in bladder cancer cells. As shown in Figure 3A, apoptotic cell death was significantly induced in 253J-BV cells exposed to diphenylene iodonium (DPI), an inhibitor of flavoprotein-dependent oxidase, or $\mathrm{N}$-acetylcysteine (NAC), an antioxidant. The induced apoptosis was further analysed by detection of PARP cleavage and DNA fragmentation (Figures $3 B$ and 3C). Consistent with the suggested role of as ROS in survival signalling in bladder cancer cells, 253J-BV cells show elevated levels of ROS that were abolished by treatment with DPI or NAC (Supplemental Figure $2 \mathrm{~B}$ ). The source of ROS generation is suspected to be via DPI-sensitive NOX, not mito- chondria, because the level of ROS was not affected by Rotenone (Supplemental Figure 2B). In addition, BLT2 inhibition by either LY255283 or knockdown of BLT2 using BLT2-specific siRNA abolished elevated ROS production (Supplemental Figures 2C and 2D). Therefore, we speculate that BLT2 promotes survival by maintaining an elevated level of ROS, possibly generated via DPI-sensitive NOX, in bladder cancer 253J-BV cells.

\section{A BLT2-NOX1/4 cascade is essential for the survival of 253J-BV bladder cancer cells}

To further elucidate the role of NOX in ROS generation in bladder cancer cells, we compared the mRNA levels of NOX family members. The 
A

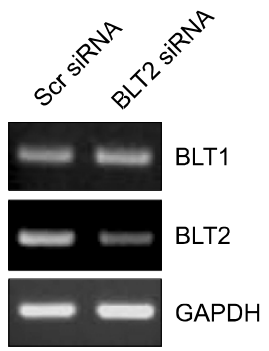

D

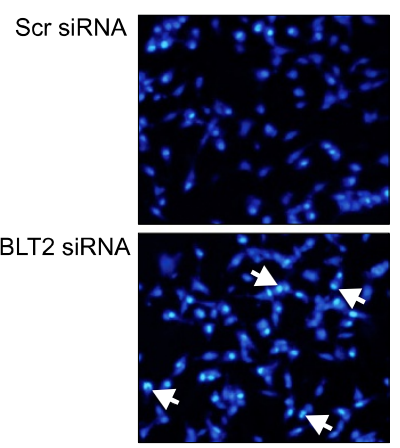

B

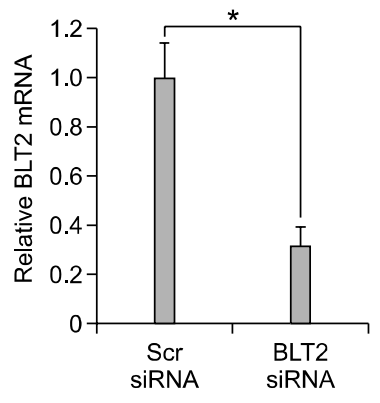

C

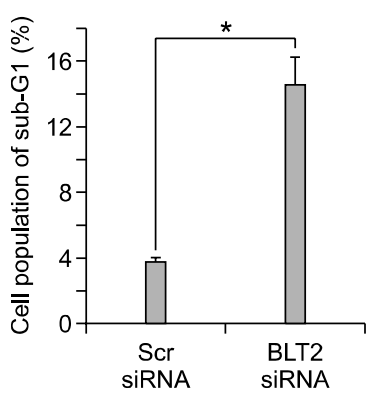

E

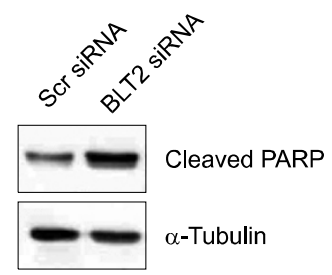

Figure 2. Induction of apoptosis by BLT2 siRNA in 253J-BV cells. (A and B) Cells were transfected with BLT2 SiRNA and, after $24 \mathrm{~h}$, the transfected cells were harvested and isolated RNA was used for semi-quantitative RT-PCR (A) and real-time PCR (B). (C) Cells were transfected with BLT2 siRNA and, after $42 \mathrm{~h}$, the sub-G1 population was determined by flow cytometric analysis. Data indicate the means \pm S.D. of three independent experiments $\left({ }^{*} P<0.01\right)$. (D) Evaluation of apoptosis by Hoechst 33258 staining as described in the Supplemental material and methods. 253J-BV cells were transfected with $50 \mathrm{nM}$ of BLT2 siRNA. After $48 \mathrm{~h}$, nuclear morphology was observed by fluorescence microscopy. Arrows indicates fragmented nuclei. (E) PARP cleavage is induced by knockdown of BLT2. Results are representative of three independent experiments with similar results. Src siRNA, Scrambled siRNA (control for BLT2 siRNA).

results show that NOX1, NOX2 and NOX4 were expressed, whereas expression of NOX5 was not detected (Supplemental Figure 3A). Among these NOX members, the levels of NOX1 and NOX4 were specifically reduced by treatment with LY255283 or knockdown of BLT2 using BLT2-specific siRNA (Supplemental Figures $3 B$ and $3 C$ ). The level of NOX2 mRNA was not affected by LY255283 or BLT2 SIRNA (data not shown). To investigate the role of NOX1 and NOX4 in cell survival, NOX1/4 knockdown was performed using pSUPER-siNOX1 and -siNOX4. The expression of endogenous NOX1 and -4 was selectively reduced upon NOX1/4specific siRNAs transfection (Figure 3D). 253J-BV cells were transfected with pSUPER-siNOX1/4 and, after $32 \mathrm{~h}$, the transfected cells were clearly reduced ROS generation (Supplemental Figure 3D). We next determined the extent of apoptosis using cell cycle analysis and pro-apoptotic protein detection. When the cells were transfected with pSUPER-siNOX1 plus -siNOX4, the proportion of sub-G1 cells and the level of apoptotic proteins (cleaved PARP) were remarkably elevated in 253J-BV cells (Figure 3E). Thus, NOX1 and NOX4dependent ROS generation, acting downstream of BLT2, appears to be critical for the survival of bladder cancer cells.

\section{A BLT2-NOX1/4 signal mediates phosphorylation of ERK/AKT}

ROS act as key mediators of MAPK and AKT signalling cascades, which regulate cell proliferation, apoptosis and cell survival (Guo et al., 2007). As shown in Figure 4A, we observed that bladder cancer cells have more activated MAPK/ERK and AKT activities than control SV-HUC-1 cells. To investigate whether activation of ERK and AKT are downstream of the 'BLT2-ROS' cascade, we measured the ability of cells were transfected with BLT2 siRNA or siNOX1/4 to affect these kinases activities in 253J-BV cells. Clearly, knockdown of the endogenous BLT2 or NOX1/4 by specific siRNAs reduced both phospho-ERK and phospho- 
A

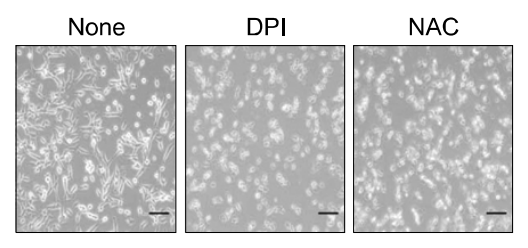

B

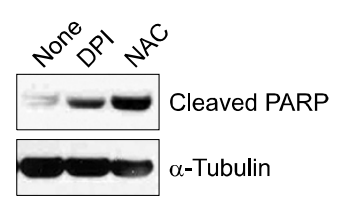

C

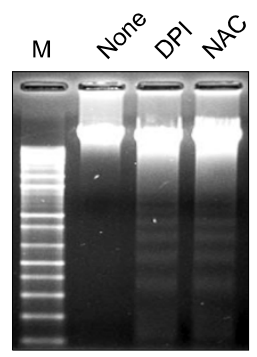

D

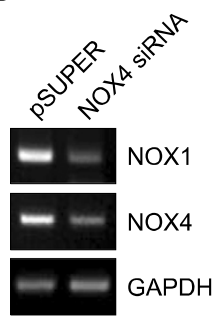

E

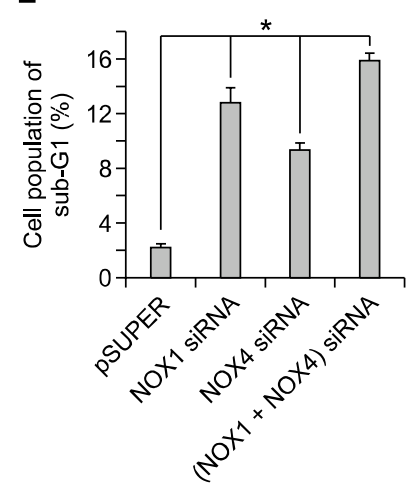

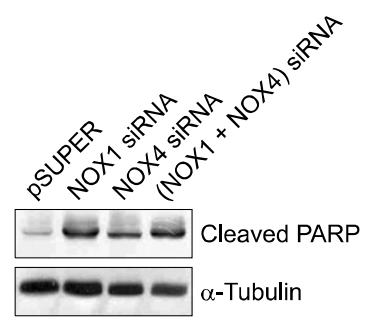

Figure 3. Induction of apoptosis by siNOX1/4 in 253J-BV cells. (A, B and C) 253J-BV cells were starved with $0.5 \%$ FBS medium for $12 \mathrm{~h}$, and then cells were treated with DPI $(5 \mu \mathrm{M})$, NAC $(10 \mathrm{mM})$ for $48 \mathrm{~h}$. (A) DPI or NAC induced cell death in 253J-BV cells. Cell morphology was visualised using an Olympus BX51 microscope at $\times 100$ magnification. Bar, $100 \mu \mathrm{m}$. (B and C) DPI and NAC induced PARP cleavage (B) and DNA laddering (C) in 253J-BV cells. The detail apoptotic analysis is described in the Supplemental material and methods. (D) The levels of NOX mRNA transcripts were measured by semi-quantitative RT-PCR at $24 \mathrm{~h}$ after transfection with pSUPER-siNOX1/4 $(1 \mu \mathrm{g})$. (E) Cell cycle arrest and PARP cleavage were induced by knockdown NOX1/4. Cells were transfected with pSUPER-siNOX1/4 $(1 \mu \mathrm{g})$ and, after $42 \mathrm{~h}$, the sub-G1 population was analysed using flow cytometry (left panel). PARP cleavage was also determined (right panel). Data indicate the means \pm S.D. of three independent experiments ( ${ }^{*} P<0.01$ vs pSUPER control vector).

AKT levels, as shown in Figures $4 B$ and $4 C$, suggesting that ERK and AKT signalling pathways are likely to mediate the survival of bladder cancer cells caused by BLT2-NOX1/4-dependent ROS production.

\section{Discussion}

Recent accumulating evidences suggest that BLT2, a receptor for $\mathrm{LTB}_{4}$ and 12(S)-HETE, is associated with various capacities of cancer cells, thus contributing to cancer progression. In this study, the role of BLT2 in the survival of bladder cancer 253J-BV cells was investigated. We observed that blockade of BLT2 with an antagonist or BLT2 siRNA results in cell cycle arrest and apoptotic cell death in human bladder cancer 253J-BV cells, suggesting a role of BLT2 in the survival of cells. Further experiments aimed at elucidating the mechanism by which BLT2 mediates survival revealed that enhanced level of ROS is generated via a BLT2-dependent up-regulation of NADPH oxidase members NOX1 and NOX4. Additionally, we observed that inhibition of ROS generation by either NOX1/4 siRNAs or treatment with an ROS-scavenging agent results in apoptotic cell death in 253J-BV bladder cancer cells. These results demonstrated that a 'BLT2NOX1/4-ROS' cascade plays a role in the survival of this aggressive bladder cancer cells, thus pointing to BLT2 as a potential target for antibladder cancer therapy.

Although, in the present study, our results point to 'survival' as a critical capacity of BLT2 in bladder cancer cells, we and others demonstrated additional novel roles of BLT2 in various cells (Choi et al., 2008; Hennig et al., 2008; Rocconi et al., 2008; Kim et al., 2009; Kim et al., 2010a; Kim and Seo et al., 2010b). Recently, we observed that the BLT2linked cascade plays a role in the invasion and metastasis of bladder cancer cells (Kim et al., 2010b), thus suggesting that BLT2 mediates at least both survival and invasion/metastasis in bladder cancer cells. Depending on experimental/ 
A

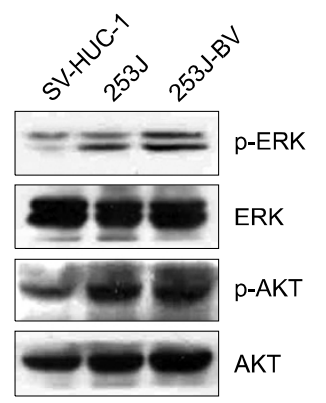

B

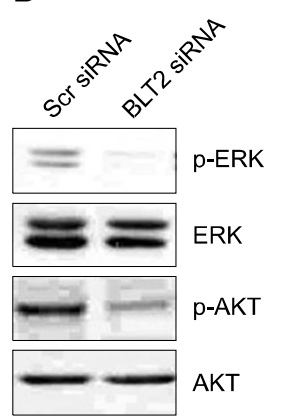

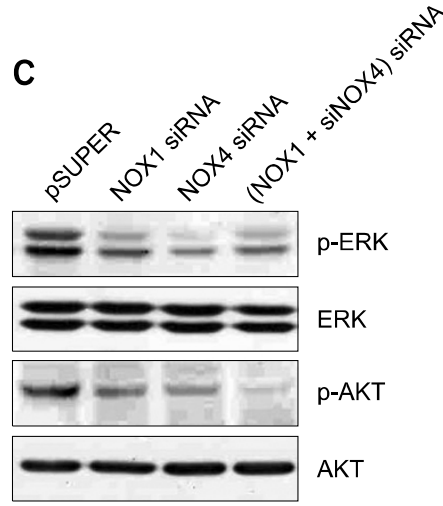

Figure 4. Effects of blockade of the BLT2-NOX signal on the ERK/AKT pathway in 253J-BV cells. (A) Relative expression of ERK and AKT in the human bladder cancer cell lines 253J and 253J-BV compared with the control SV-HUC-1. (B) Knockdown of BLT2 by specific siRNA reduced phosphorylation of ERK and AKT in 253J-BV cells. Cells were transfected with BLT2 siRNA ( $50 \mathrm{nM}$ ) for $36 \mathrm{~h}$ and proteins were separated by SDS-PAGE (8\%), transferred onto PVDF membranes, and detected with specific antibodies. (C) Knockdown of NOX1/4 reduced phosphorylation of ERK and AKT in 253J-BV cells. pSUPER and pSUPER-siNOX1/4 $(1 \mu \mathrm{g})$ were transiently transfected into 253J-BV cells. After $36 \mathrm{~h}$, the cells were harvested for detection of ERK and AKT with specific antibodies. Results are representative of three independent experiments with similar results.

culture conditions, each specific capacities of BLT2 are likely to be preferentially demonstrated (e.g., survival phenotype in serum-deprived conditions; invasion phenotype in serum-rich culture conditions). Thus, we suspect that culture conditions somehow contribute to differential capacities of BLT2 and further study is needed to elucidate the detailed underlying mechanisms by which capacities of BLT2 are regulated.

In general, moderate levels of ROS play important roles in cell proliferation and survival, whereas severe increases or decreases of ROS can induce cell death (Trachootham et al., 2008). Cancer cells often exhibit increased production of ROS compared with control cells. Intrinsic ROS in cancer cells seem to provide the cells with a survival advantage. Similarly, we also observed that most bladder cancer cells show enhanced ROS generation compared with control bladder cells (Supplemental Figure 2A). In bladder cancer, it was recently reported that the acquisition of metastatic ability is associated with the level of ROS scavenging enzymes in the bladder cancer cell lines 253J (non-metastatic) and 253J-BV (highly metastatic) (Hempel et al., 2009). However, no direct evidence that NOX-derived ROS are responsible for cell survival has been reported in bladder cancer cells although recently we observed that BLT2-linked ROS generation by NOX1 and NOX4 plays a role of bladder cancer invasion (Kim et al., 2010b). Using siRNAs specific for NOX1 and NOX4, we observed that knockdown of NOX1/4 had a significant inhibitory effect on bladder cancer cell survival, leading to apoptosis, suggesting that
ROS produced by NOX1/4 exert anti-apoptotic function (Figure 3 ). In agreement with our findings, Lee et al. reported that ROS produced by NOX4 inhibit apoptosis in pancreatic cancer MiaPaCa cells (Lee et al., 2007). In addition, our recent data showed the elevated ROS level is through a 'BLT2-NOX1'-linked cascade in breast cancer cells and is involved in the pro-survival, especially in ER-negative breast cancer cells (Choi et al., 2010). Therefore, taken together with the previous study results, ROS, especially BLT2-NOX-derived ROS, may play quite general role in mediating the survival of various cancer cells. Although we did not examine the mechanism of the survival role of NOX-derived ROS, ROS have been reported to inhibit tyrosine phosphatases (PTPs) and thus could sustain the activation of kinases, eventually leading to cell growth and survival (Lee et al., 2007; Trachootham et al., 2008). Alternatively, the pro-survival and growth effects of ROS could be mediated via anti-apoptotic pathways, such as those involving ERK and AKT (Mochizuki et al., 2006; Rygiel et al., 2008). In accordance with these reports, we observed that the levels of phosphorylated ERK and AKT are higher in 253J-BV cells compared with control cells, and following transfection with BLT2 siRNA or siNOX1/4, phosphorylation of ERK and AKT was clearly reduced in 253J-BV cells (Figure 4), thus suggesting that 'BLT2-NOX1/4-ROS'- dependent cell survival signalling is, at least in part, mediated by ERK/AKT cascade. Further studies elucidating the linkage between ROS and ERK/ AKT will likely be pivotal to our better understanding of BLT2-mediated signalling 
to survival in bladder cancer cells.

Our findings that a 'BLT2-NOX1/4-ROS' cascade is associated with the survival of bladder cancer cells will provide valuable information on the mechanism of bladder cancer progression and contribute to the development of anti-bladder cancer therapy.

\section{Methods}

\section{Cell culture and materials}

SV-HUC-1 immortalised human uroepithelial cells were obtained from the American Type Culture Collection (ATCC, Rockville, MD) and maintained in F-12 Kaighn's medium (GIBCO; Grand Island, NY) containing 10\% FBS The human bladder cancer cell lines HT-1376, 253J, J82, T24 (human bladder carcinoma) and 253J-BV (human bladder transitional cell carcinoma) were obtained from the American Type Culture Collection (ATCC, Rockville, MD), and cells were maintained in DMEM or RPMI 1640 supplemented with $10 \%$ FBS and antibiotic-antimycotic solution (Life Technologies, Gaithersburg, MD) at $37^{\circ} \mathrm{C}$ in a humidified atmosphere of $5 \% \mathrm{CO}_{2}$.

Dimethyl sulfoxide (DMSO) was obtained from SigmaAldrich (St. Louis, MO). The BLT2 antagonist LY255283 was purchased from Cayman Chemical Company (Ann Arbor, MI) and the BLT1 antagonist U75302 was from BioMol (Plymouth Meeting, PA). DPI, Rotenone and NAC were obtained from Calbiochem (La Jolla, CA), and the $\mathrm{H}_{2} \mathrm{O}_{2}$-sensitive fluorophore $2^{\prime}, 7^{\prime}$-dichlorofluorescein diacetate (DCF-DA) was from Molecular Probes (Eugene, OR). Fetal bovine serum (FBS), Dulbecco's modified Eagle's medium (DMEM), RPMI 1640 and nonessential amino acids were from Life Technologies, Inc. (Gaithersburg, MD). All other chemicals were from standard sources and were of molecular biology grade or higher.

\section{Cell cycle analysis}

Sub- $G_{1}$ apoptotic populations were assessed as described previously (Choi et al., 2010). In brief, 253J-BV cells were seeded in 6 -well at $2.5 \times 10^{5}$ cells and allowed to grow for $24 \mathrm{~h}$. For cell cycle analysis, cells were starved with $0.5 \%$ FBS medium for $12 \mathrm{~h}$. The cells were treated with $10 \mu \mathrm{M}$ LY255283 or $1 \mu \mathrm{M}$ U75302 for $42 \mathrm{~h}$ in $0.5 \%$ FBS medium before analysis. For siRNA experiments, cells were transfected with siRNA and, after $42 \mathrm{~h}$, the sub- $\mathrm{G}_{1}$ population was analyzed by FACS. Acquisition and analysis was performed by FACS using Cell Quest Alias software (BD Bioscience).

\section{Cell viability}

Cell viability was detected by MTT assay at $48 \mathrm{~h}$. In brief, $1 \times 10^{4}$ cells per well were plated in a 96-well plates in culture complete medium. Cells were incubated with $0.5 \%$ FBS medium for $12 \mathrm{~h}$. And then cells were treated with indicated antagonists. After $48 \mathrm{~h}$, cells were washed with PBS and MTT solution ( $5 \mathrm{mg} / \mathrm{L}$, obtain from Sigma, St
Louis, MO) was added to each well and incubated at $37^{\circ} \mathrm{C}$ for $4 \mathrm{~h}$. The culture medium was removed and $100 \mu \mathrm{l}$ of DMSO was added to dissolve the formazan. Finally, the density of formazan in each well was detected at $590 \mathrm{~nm}$ using a microplate reader.

\section{DNA fragmentation assay}

Cells were incubated in medium containing $0.5 \%$ FBS 12 h. Then, cells were treated with LY255283 (10 $\mu \mathrm{M})$, U75302 $(1 \mu \mathrm{M})$, DPI $(5 \mu \mathrm{M})$ or NAC $(10 \mathrm{mM})$ for $48 \mathrm{~h}$. Cells were collected and resuspended in a lysis buffer $[20 \mathrm{mM}$ Tris- $\mathrm{HCl}$ ( $\mathrm{pH} 8.0), 0.1 \mathrm{mM}$ ethylenediaminetetraacetic acid, $1 \%$ sodium dodecyl sulfate and $0.5 \mathrm{mg} / \mathrm{ml}$ proteinase $\mathrm{K}]$ and then incubated at $50^{\circ} \mathrm{C}$ overnight. DNA was extracted with phenol-chloroform. DNA samples were electrophoresed on a $1.8 \%$ agarose gel.

\section{Hoechst staining}

253J-BV cells $\left(2 \times 10^{5}\right)$ were plated, allowed to attach overnight, and cells were transfected with $50 \mathrm{nM}$ of siBLT2 for $48 \mathrm{~h}$. For Hoechst 33258 staining, $5 \mu \mathrm{g} / \mathrm{ml}$ of Hoechst 33258 (Sigma, St Louis, MO) was added to $35 \mathrm{~mm}$ dishes and incubated in the dark for $15 \mathrm{~min}$ at $37^{\circ} \mathrm{C}$. Cells were scored blindly for apoptotic morphological features using fluorescence microscopy (Carl Zeiss, Germany). Bright blue spots indicating nuclear condensation and fragmentation were counted in three random fields.

\section{Quantitative and real-time PCR analysis}

Total cellular RNA was extracted from cells using Easy-Blue from Intron Co. (Daejeon, Korea). First-strand cDNA was prepared in buffer containing $0.5 \mu \mathrm{g}$ of oligo $(\mathrm{dT})_{15}$ primer, $10 \mathrm{mM}$ dithiothreitol, $0.5 \mathrm{mM}$ dNTP mix (TaKaRa), and 200 units of M-MLV Reverse Transcriptase and incubated at $37^{\circ} \mathrm{C}$ for $50 \mathrm{~min}$. The primer sequences used are as follows: human BLT1 (forward, 5'-TATGTCT GCGGAGTCAGCATGTACGC-3'; reverse, 5'-CCTGTAG CCGACGCCCTATGTCCG-3'); human BLT2 (forward, 5'AGCCTGGAGACTCTGACCGCTTTCG-3'; reverse, 5'-GA CGTAGCACCGGGTTGACGCTA-3'); human NOX4 (forward, 5'-CTCAGCGGAATCAATCAGCTGTG-3'; reverse, 5'-AGAGGAACACGACAATCAGCCTTAG-3'); and GAPDH (forward, 5'-CTGCACCACCAACTGCTTAGC-3'; reverse, $5^{\prime}$-CTTCACCACCTTCTTGATGTC-3'). The analysis of NOX1 mRNA was performed by two-step RT-PCR as described previously (Chamulitrat et al., 2003). For the first-round PCR, (forward, 5'-CAGGGAGACAGGTGCCTT TTCC-3'; reverse, 5'-GCTCAAACCTGACGAGACCAAG-3') were used, and for the second-round nested PCRs, (forward, 5'-AACCTGTTGACTTCCCTGGAAC-3'; reverse, 5'-TCCAGACTGGAATATCGGTGAC-3') were used. PCR products were purified by $1.5 \%$ agarose gel electrophoresis and visualised with ethidium bromide. For realtime PCR, data were analysed with LightCycler software 3.3 (Roche Diagnostics, Indianapolis, IN). The primer sequences used for BLT1 and BLT2 are (forward, 5'CCTGAAAAGGATGCAGAAGC-3'; reverse, 5'-AAAAAGG GAGCAGTGAGCAA-3') and (forward, 5'-CTTCTCATCGG GCATCACAG-3'; reverse, 5'-ATCCTTCTGGGCCTACAGGT- 
3'), respectively.

\section{Preparation of cell extracts and western blotting}

Cells were washed with cold PBS and scraped into lysis buffer [20 mM Tris- $\mathrm{HCl}$ (pH 7.5), $150 \mathrm{mM} \mathrm{NaCl}, 0.5 \%$ NP-40, $5 \mathrm{mM}$ EDTA, 1\% Triton X-100 and protease inhibitors] at $4^{\circ} \mathrm{C}$. Harvested protein samples were heated at $95^{\circ} \mathrm{C}$ for $5 \mathrm{~min}$ and then subjected to SDS-PAGE in acrylamide gels, followed by transfer to polyvinylidene difluoride (PVDF) membranes for $90 \mathrm{~min}$ at $100 \mathrm{~V}$. The membranes were blocked for $1 \mathrm{~h}$ with Tris-buffered saline (TBS) containing $0.05 \%$ ( $\mathrm{vol} / \mathrm{vol}$ ) Tween 20 plus $5 \%$ (wt/vol) nonfat dry milk and then incubated with the appropriate antibodies. Poly ADP-ribose polymerase (PARP), caspase-9, Bax and MAP Kinases antibodies were obtained from Cell Signaling Technology (Danvers, MA) (PARP, 1:2000 dilution; Caspase-9, 1:2000 dilution; Bax, 1: 2000 dilution; MAP kinases, 1:2000 dilution; actin, 1:3000 dilution). Primary antibodies in $5 \%$ nonfat milk overnight at $4^{\circ} \mathrm{C}$. The membrane-bound protein-antibody complexes were then incubated for $2 \mathrm{~h}$ with horseradish peroxidise (HRP)-conjugated secondary antibody before development with an enhanced chemiluminescence kit (Amersham Biosciences, UK).

\section{Cell transfection with siRNA}

Scrambled and BLT2-specific siRNA (5'-CCACGCAGTC AACCTTCTG-3') were purchased from Bioneer (Daejeon, Korea) (Hennig et al., 2008). The mammalian expression vector pSUPER (scrambled vector as a control) (OligoEngine) was used to express the siRNA. pSUPER vector (control), pSUPER-siNOX1 and pSUPER-siNOX4 were provided by Dr. Yoon-Soo Bae at Ewha's Women University (Seoul, Korea), and the NOX1/4 target sequences were previously described (Park et al., 2004). BLT2 siRNA oligonucleotides and pSUPER-siNOX1 and -siNOX4 were introduced into the cells using Lipofectamine (Invitrogen, Calsbad, CA), according to the manufacturer's instructions. Cells were incubated for $24 \mathrm{~h}$, and then the culture medium was changed to complete medium. At the indicated time point, the transiently transfected cells were harvested for RNA and protein extraction.

\section{Data analysis and statistics}

Data are expressed as mean percentages of the control \pm S.D. One-way analysis of variance ANOVA was used for comparison of multiple groups. Groups were compared by Student's $t$ test, and values of $P<0.05$ were considered significant.

\section{Supplemental data}

Supplemental data include three figures and can be found with this article online at http://e-emm.or.kr/article/article files/SP-43-3-01.pdf.

\section{Acknowledgements}

This work was supported by a General Researcher Support Project (2010-0008295), and a Diseases Network Research Program grant from the Ministry of Education, Science and Technology, Republic of Korea. In addition, this work was supported by a grant of the Korea Healthcare technology R\&D Project, Ministry of Health and Welfare, Republic of Korea (A101032) and a grant from KIST Institutional R\&D Program.

\section{References}

Chamulitrat W, Schmidt R, Tomakidi P, Stremmel W, Chunglok W, Kawahara T, Rokutan K. Association of gp91phox homolog Nox1 with anchorage-independent growth and MAP kinase-activation of transformed human keratinocytes. Oncogene 2003;22:6045-53

Choi JA, Kim EY, Song H, Kim C, Kim JH. Reactive oxygen species are generated through a BLT2-linked cascade in Ras-transformed cells. Free Radic Biol Med 2008;44:624-34

Choi JA, Lee JW, Kim H, Kim EY, Seo JM, Ko J, Kim JH. Pro-survival of estrogen receptor-negative breast cancer cells is regulated by a BLT2-reactive oxygen species-linked signaling pathway. Carcinogenesis 2010;31:543-51

Cordon-Cardo C. Molecular alterations associated with bladder cancer initiation and progression. Scand J Urol Nephrol Suppl 2008;218:154-65

Guo J, Zhu T, Xiao ZX, Chen CY. Modulation of intracellular signaling pathways to induce apoptosis in prostate cancer cells. J Biol Chem 2007;282:24364-72

Hayashi T, Nishiyama K, Shirahama T. Inhibition of 5-lipoxygenase pathway suppresses the growth of bladder cancer cells. Int J Urol 2006;13:1086-91

Hempel N, Ye H, Abessi B, Mian B, Melendez JA. Altered redox status accompanies progression to metastatic human bladder cancer. Free Radic Biol Med 2009;46:42-50

Hennig R, Ding XZ, Tong WG, Witt RC, Jovanovic BD, Adrian TE. Effect of LY293111 in combination with gemcitabine in colonic cancer. Cancer Lett 2004;210:41-6

Hennig R, Osman T, Esposito I, Giese N, Rao SM, Ding XZ, Tong WG, Buchler MW, Yokomizo T, Friess H, Adrian TE. BLT2 is expressed in PanINs, IPMNs, pancreatic cancer and stimulates tumour cell proliferation. Br J Cancer 2008;99: 1064-73

Jemal A, Siegel R, Ward E, Hao Y, Xu J, Murray T, Thun MJ. Cancer statistics, 2008. CA Cancer J Clin 2008;58:71-96

Kaufman DS, Shipley WU, Feldman AS. Bladder cancer. Lancet 2009;374:239-49

Kim EY, Seo JM, Cho KJ, Kim JH. Ras-induced invasion and metastasis are regulated by a leukotriene B4 receptor BLT2-linked pathway. Oncogene 2010a;29:1167-78

Kim EY, Seo JM, Kim C, Lee JE, Lee KM, Kim JH. BLT2 promotes the invasion and metastasis of aggressive bladder cancer cells through a reactive oxygen species-linked pathway. Free Radic Biol Med 2010b;49:1072-81 
Kim GY, Lee JW, Cho SH, Seo JM, Kim JH. Role of the low-affinity leukotriene B4 receptor BLT2 in VEGF-induced angiogenesis. Arterioscler Thromb Vasc Biol 2009;29:91520

Lee JK, Edderkaoui M, Truong P, Ohno I, Jang KT, Berti A, Pandol SJ, Gukovskaya AS. NADPH oxidase promotes pancreatic cancer cell survival via inhibiting JAK2 dephosphorylation by tyrosine phosphatases. Gastroenterology 2007;133:1637-48

Mochizuki T, Furuta S, Mitsushita J, Shang WH, Ito M, Yokoo Y, Yamaura M, Ishizone S, Nakayama J, Konagai A, Hirose K, Kiyosawa K, Kamata T. Inhibition of NADPH oxidase 4 activates apoptosis via the AKT/apoptosis signal- regulating kinase 1 pathway in pancreatic cancer PANC-1 cells. Oncogene 2006;25:3699-707

Nie D, Krishnamoorthy S, Jin R, Tang K, Chen Y, Qiao Y, Zacharek A, Guo Y, Milanini J, Pages G, Honn KV. Mechanisms regulating tumor angiogenesis by 12-lipoxygenase in prostate cancer cells. J Biol Chem 2006;281:18601-19

Park HS, Lee SH, Park D, Lee JS, Ryu SH, Lee WJ, Rhee SG, Bae YS. Sequential activation of phosphatidylinositol 3-kinase, beta Pix, Rac1, and Nox1 in growth factor-induced production of H2O2. Mol Cell Biol 2004;24:4384-94

Rocconi RP, Kirby TO, Seitz RS, Beck R, Straughn JM Jr, Alvarez RD, Huh WK. Lipoxygenase pathway receptor expression in ovarian cancer. Reprod Sci 2008;15:321-6

Rygiel TP, Mertens AE, Strumane K, van der Kammen R, Collard JG. The Rac activator Tiam1 prevents keratinocyte apoptosis by controlling ROS-mediated ERK phosphorylation. J Cell Sci 2008;121:1183-92
Tager AM, Luster AD. BLT1 and BLT2: the leukotriene B(4) receptors. Prostaglandins Leukot Essent Fatty Acids 2003;69:123-34

Tong WG, Ding XZ, Talamonti MS, Bell RH, Adrian TE. LTB4 stimulates growth of human pancreatic cancer cells via MAPK and PI-3 kinase pathways. Biochem Biophys Res Commun 2005;335:949-56

Tong WG, Ding XZ, Talamonti MS, Bell RH, Adrian TE. Leukotriene B4 receptor antagonist LY293111 induces S-phase cell cycle arrest and apoptosis in human pancreatic cancer cells. Anticancer Drugs 2007;18:535-41

Trachootham D, Lu W, Ogasawara MA, Nilsa RD, Huang P. Redox regulation of cell survival. Antioxid Redox Signal 2008;10:1343-74

Woo CH, You HJ, Cho SH, Eom YW, Chun JS, Yoo YJ, Kim $\mathrm{JH}$. Leukotriene $\mathrm{B}(4)$ stimulates Rac-ERK cascade to generate reactive oxygen species that mediates chemotaxis. J Biol Chem 2002;277:8572-8

Wu WS. The signaling mechanism of ROS in tumor progression. Cancer Metastasis Rev 2006;25:695-705

Yoshimura R, Matsuyama M, Tsuchida K, Kawahito Y, Sano $\mathrm{H}$, Nakatani T. Expression of lipoxygenase in human bladder carcinoma and growth inhibition by its inhibitors. J Urol 2003;170:1994-9

Zhang W, McQueen T, Schober W, Rassidakis G, Andreeff M, Konopleva M. Leukotriene B4 receptor inhibitor LY293111 induces cell cycle arrest and apoptosis in human anaplastic large-cell lymphoma cells via JNK phosphorylation. Leukemia 2005;19:1977-84 